\title{
Local Resection of Rectal Cancer after Neoadjuvant Therapy: A New Trend for Organ Preservation
}

\author{
Carlos Casimiro da Costa Pereira ${ }^{1}$, Olga Manuela Duarte Correia de Oliveira ${ }^{1}$, \\ Nuno Miguel Afonso Vilela Pinto ${ }^{1}$, Sandra Fátima Fernandes Martins ${ }^{1,2}$, \\ Fernanda Maria da Costa Nogueira ${ }^{1}$, Joaquim Manuel da Costa Pereira ${ }^{1}$
}

'General Surgery Department, Hospital de Braga, Braga, Portugal

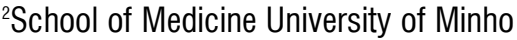

\section{ABSTRACT}

Low anterior resection for rectal cancer is associated with high morbidity, permanent stomas and functional impairment. Different strategies to avoid this surgery in low rectal cancer are being pursued. Several prospective trials have explored a strategy of neoadjuvant treatment to downstage rectal cancer and allow for local resection of the tumor. In this paper we review these trials and the published results of this strategy in regards of patient selection, successful downstaging, oncologic outcomes, morbidity and functional outcomes. In selected patients, this new strategy achieved good downstaging of tumors and similar oncologic outcomes when compared to radical resection. Some questions regarding patient selection remain, mainly which patients should undergo completion surgery after local resection.

Key words: early rectal cancer, local resection of rectal cancer, neoadjuvant treatment

\section{INTRODUCTION}

The concept of organ preservation in rectal cancer surgery is extremely appealing for mainly two reasons. First, low anterior resection surgery is associated with a high morbidity rate and permanent stomas. Second, it is also associated with functional impairment with low anterior resection syndrome (LARS), sexual and urinary problems and fecal incontinence. The preservation of the rectum could avoid this morbidity. Currently two strategies allow for rectal preservation in rectal cancer, local resection and watch and wait protocol. Without any other kind of treatment, the former is appropriate only to pT1 rectal cancer, while the latter involves neoadjuvant treatment to achieve a complete clinical response.

In this paper we sought to review the literature of a different strategy, neo-adjuvant treatment to downstage rectal cancer and allow for a local resection.
Corresponding author: Carlos Casimiro da Costa Pereira Serviço de Cirurgia Geral Hospital de Braga

Sete Fontes - Săo Victor 4710-243

Braga, Portugal

Phone: +351933320148

E-mail: carlos.pereira@hb.min-saude.pt 


\section{The paradox of current rectal cancer management}

Current rectal cancer treatment is somewhat paradoxical. Early stage rectal cancer (pT1) is amenable to local excision. Locally advanced rectal cancer is treated with neoadjuvant therapy first (1) but in the setting of a complete clinical response some groups use a watch and wait strategy and the patient does not even undergo surgery (2). In T2NO rectal cancer the only accepted strategy is radical surgery (1), which means that earlier rectal cancer is treated in a potentially more aggressive way than more advanced rectal cancer. Using neoadjuvant treatment to downstage $\mathrm{T} 2$ rectal cancer may be an answer to try to spare patients major surgery. In the setting of a noncomplete response to neoadjuvant treatment local excision might be enough because of tumor downstaging and sterilization of the mesorectum.

\section{Prospective studies}

There are two prospective cohorts and two randomized controlled trials (RCT) investigating the downstaging of rectal tumors with neoadjuvant chemoradiation followed by transanal excision (table 1). All these studies selected patients with small tumors in the mid or low rectum in which local excision was feasible.

The earliest of these trials was a RCT published in 2012 by Lezoche et al (3). It involved two Italian centers. In total, 102 patients with rectal cancer staged T2, $\mathrm{G} 1 / \mathrm{G} 2$, under $3 \mathrm{~cm}$ diameter and no lymphovascular or perineural invasion were randomized after neoadjuvant chemoradiation to either local excision or total mesorectal excision (TME).

Two prospective cohorts were published in 2015. The ACOZOG Z6041 (4) was a multicentric prospective trial in which patients with clinical T2NO rectal cancer, under $4 \mathrm{~cm}$ diameter and less than $40 \%$ of the rectal circumference were given neoadjuvant chemoradiation with capecitabine and oxaliplatin followed by transanal excision. Patients with positive margins or ypT3 tumors after local excision were offered radical surgery. As in Lezoche's study yрT2 patients entered follow-up. Of the 79 patients recruited, two did not undergo surgical treatment due to neoadjuvant treatment related complications, one had a TME and an additional four did not fulfill the criteria of rectal preservation.

CARTS (5) was a prospective multicenter cohort which differs from the previous study in several important aspects. First, it enrolled patients with a clinical stage T1-T3 NO. Second, it used the tumor response to chemoradiation to select patients for local resection. Third, patients with > ypT1 tumors were subject to major surgery after local excision. It enrolled 55 patients but because of neoadjuvant treatment complications 4 patients did not undergo surgical treatment. In $9 \%$ of the patients enrolled MRI revision reclassified the disease as $\mathrm{N} 1$.

The GRECCAR 2 (6) study was a prospective multicentric randomized controlled trial that randomized 145 patients with cT2-T3 rectal cancer with a maximum diameter of $4 \mathrm{~cm}$ and with good clinical response to neoadjuvant chemoradiation defined as residual tumor under $2 \mathrm{~cm}$. It allowed inclusion of patients with up to three positive lymph nodes in the clinical staging. The primary endpoint of this study was a composite score of death, recurrence, major surgical morbidity and severe complications. Of the 186 patients recruited, 148 were considered good responders to chemoradiotherapy and ultimately 145 were randomized to either local excision or TME. There was a significant proportion of patients randomized to TME that did not undergo that surgery, 8 received local excision and 3 received no surgery.

\section{Downstaging of tumors}

The downstaging after neoadjuvant treatment,

Table 1 - Summary of study characteristics and inclusion criteria

\begin{tabular}{|c|c|c|c|c|c|c|}
\hline \multicolumn{7}{|c|}{ Patient Selection } \\
\hline Study & Year & Type & Staging & Features & Restaging & $\begin{array}{l}\text { Completion } \\
\text { TME }\end{array}$ \\
\hline CARTS & 2015 & Prospective Cohort & cT1-3 N0 & up to $10 \mathrm{~cm}$ anal verge & cT0-2 & $>\mathrm{ypT1}$ \\
\hline $\begin{array}{l}\text { ACOZOG } \\
\text { Z6041 }\end{array}$ & 2015 & Prospective Cohort & cT2 NO & $\begin{array}{l}<4 \mathrm{~cm} \text { diameter, }<40 \% \text { rectal circumference, } \\
<8 \mathrm{~cm} \text { from the anal verge }\end{array}$ & No influence & > ypT2 \\
\hline Lezoche & 2012 & RCT & CT2 NO & $<3 \mathrm{~cm},<6 \mathrm{~cm}$ from the anal verge, $\mathrm{G} 1$ or 2 & No influence & none \\
\hline GRECCAR 2 & 2017 & RCT & cT2-3 N0-1 & $<4 \mathrm{~cm},<8 \mathrm{~cm}$ from the anal verge & $\begin{array}{l}\text { good clinical } \\
\text { response }^{1}\end{array}$ & $>$ ypT1 \\
\hline
\end{tabular}

${ }^{1}$ good clinical response defined as a scar with less than $2 \mathrm{~cm}$ and no vegetative component 
morbidity and oncologic results are summarized in tables 2 and 3.

In Lezoche's study about half the patients were downstaged to ypT1 or ypT0 and around half of the patients that remained $\mathrm{T} 2$ had downsize of at least 50\% of tumor volume. They reported no ypT3 tumors. No patients in the local excision group had a completion TME. In Z6041 major surgery was offered to patients with positive margins or ypT3 histology. Almost half of the patients achieved a complete pathologic response, 14\% had ypT1, 31\% ypT2, 4\% ypT3 and 1 patient ypTx. In the intention to treat group, at the end of follow-up, $91 \%$ of patients that received neoadjuvant chemoradiation fulfilled the criteria for rectal preservation.

In the CARTS study, of the 55 patients included only 47 were restaged ypT0-2 after neoadjuvant chemoradiotherapy and proceeded to local resection. In only 30 patients ( $55 \%$ of the sample) the criteria established for rectal preservation after local excision was achieved. In this study the criteria for rectal preservation was stricter than the previous referred studies. It did not allow for rectal preservation in ypT2 tumor. If the criteria of the previous studies were followed a further 15 patients, with ypT2 tumors, would have had rectal preservation bringing the total up to $81,8 \%$ which is not very different from the $Z 6041$ study. If we applied the CARTS criteria to Lezoche's and Z6041 studies the rate of rectal preservation would be $52 \%$ and $63 \%$ respec- tively. Like the CARTS study, in GRECCAR 2, ypT0-1 were followed-up, ypT2-3 and R1 had a TME. In the complete cohort $61 \%$ were deemed to have a good pathological response (57 patients (40\%) ypT0 and $29(20 \%)$ ypT1), 44 patients (31\%) were ypT2 and 12 (9\%) were ypT3. Again, this distribution is very similar to the previous studies.

\section{Oncologic outcomes}

Both RCT found no difference in local recurrence, distant metastasis, overall survival and disease-free survival between local excision and TME. All studies have different follow-up times, but local recurrence is between 4 to $8 \%$. After local excision, local recurrences tend to happen within the first year of follow-up. Lezoche does not describe the salvage treatment after local recurrence. In the other studies 9 of 12 patients with local recurrence underwent salvage surgery.

Distant metastasis occurred in 4 to $8 \%$ in most studies but in GRECCAR 2 distant metastasis rate was $15 \%$ in local excision group and $13 \%$ in the TME group. In this study, $38 \%$ of the recruited patients had N1 disease which might explain the higher rate of distant metastasis. Overall survival is between $72 \%$ at almost 10 years and $82,8 \%$ at 53 months. Disease-free survival ranges from $75 \%$ to $89 \%$. Again, in GRECCAR 2 the disease-free survival is lower than the observed in

Table 2 - Final pathology after neoadjuvant treatment

\begin{tabular}{|c|c|c|c|c|c|c|c|c|}
\hline & Lezoche & & CARTS & & Z6041 & & \multicolumn{2}{|c|}{ GRECCAR2 } \\
\hline patients with surgery & 100 & & 47 & & 77 & & 142 & \\
\hline урт0 & 27 & $27.0 \%$ & 21 & $44.7 \%$ & 38 & $49.4 \%$ & 57 & $40.1 \%$ \\
\hline урт1 & 24 & $24.0 \%$ & 9 & $19.1 \%$ & 11 & $14.3 \%$ & 29 & $20.4 \%$ \\
\hline урТ2 & 49 & $49.0 \%$ & 15 & $31.9 \%$ & 24 & $31.2 \%$ & 44 & $31.0 \%$ \\
\hline урт3 & 0 & $0.0 \%$ & 1 & $2.1 \%$ & 3 & $3.9 \%$ & 12 & $8.5 \%$ \\
\hline урТх & & & & & 1 & $1.3 \%$ & & \\
\hline
\end{tabular}

Table 3 - Morbidity and oncologic results

\begin{tabular}{lcccccc}
\hline & \multicolumn{2}{c}{ Lezoche } & Z6041 & CARTS & \multicolumn{2}{c}{ GRECCAR 2 } \\
\hline & LE & TME & & & LE & TME \\
\hline $\mathrm{n}$ & 50 & 50 & 79 & 47 & 81 & 71 \\
\hline Follow-up time & 9.6 years & & 56 months & 53 months & 3 years & \\
\hline Surgical morbidity & $2 \%$ & $6 \%$ & $2 \%$ & $10.6 \%$ & $23 \%$ & $21 \%$ \\
\hline Local recurrence & $8 \%$ & $6 \%$ & $4 \%$ & $7.80 \%$ & $6 \%$ & $3 \%$ \\
\hline Distant metastasis & $4 \%$ & $4 \%$ & $6 \%$ & $7.80 \%$ & $15 \%$ & $13 \%$ \\
\hline Overall survival & $72 \%$ & $80 \%$ & $94.8 \%$ & $82.8 \%$ & $89 \%$ & $95 \%$ \\
\hline Disease free survival & & & $88 \%$ & $81.6 \%$ & $75 \%$ & $82 \%$ \\
\hline
\end{tabular}


other studies probably because of the types of patients recruited. All authors consider local excision after neoadjuvant treatment in selected patients to be oncologic safe, either because their own study shows no difference in the outcomes between groups or their results are similar to results previously reported in total mesorectal excision for rectal cancer.

\section{Morbidity}

Local excision seems to be an excellent alternative to total mesorectal excision to decrease de morbidity of surgery. There is no risk of anastomotic leak with peritonitis, and most morbidity after local excision is minor (7). Local excision after neoadjuvant chemoradiation might have higher morbidity (8). Lezoche found no difference in morbidity between local excision and total mesorectal excision, with a rate of major morbidity 2 and $6 \%$ respectively. They found differences in the stoma rate, temporary and definitive, duration of surgery, blood loss and need for transfusion.

In CARTS five major complications were identified and 4 needed reoperation, one rectovaginal fistula, one hemorrhage and two pre-sacral abscess that required a colostomy formation.

In GRECCAR 2 local excision had a rate of major complications of $11 \%$ (6/53 patients) while the TME group had a rate of $21 \%$ (13/61 patients).

Chemoradiotherapy is also an important source of morbidity. Due to unfavorable toxic effects the chemoradiation protocol of Z6041 study had to be revised. Originally, the protocol used capecitabine and oxaliplatin in addition to radiation. The radiation and capecitabine dose were reduced.

In GRECCAR 2 the chemotherapy regimen was also changed. The use of oxaliplatin was discontinued because of the results of a phase III trial (9) and indeed they found lower side effects in patients with the revised protocol.

In CARTS two patients died because of chemoradiation therapy complications and another two had severe side effects that precluded them to have surgery, another patient in Z6041 had no surgery because of chemotherapy side effects. Most of the patients recruited in these studies would have not had neoadjuvant treatment for their tumors (<T3 NO).

\section{Who could benefit from this strategy}

Even though GRECCAR 2 found no difference in oncologic outcomes between the two strategies, they were not able to affirm the superiority of the local excision strategy. This happened because although the surgical complications of the local excision group were less severe than the TME group, the completion TME after local excision group had more than twice the complication rate of the TME group ( 21 vs $46 \%$ ). The high rate of complications is this subgroup offset the advantaged of local excision so there was no difference in the composite outcome between groups.

This is an extremely interesting point, the strategy of neoadjuvant therapy followed by local excision seems to be a tradeoff between surgical morbidity of TME and chemoradiation morbidity and the possibility of increased morbidity with completion TME after local excision. The key seems to be patient selection. Using the clinical response to the neoadjuvant therapy does not seem to have a very good correlation with the final pathological outcome. Even in good responders around $30 \%$ of patients have at least ypT2 tumors and $10 \%$ have ypT3 tumors. Considering the results of these trials the question is if we can preserve the rectum in ypT2 patients.

Both Z6041 and Lezoche's studies did not perform completion TME in ypT2 patients. Lezoche does not detail in whom recurrence occurred. In $Z 6041$ two recurrences in 24 patients with ypT2 were detected, one local and one lung metastasis, an $8,3 \%$ rate. In contrast, CARTS study found three local recurrences in 9 patients with ypT2 histology that refused further surgery. Even accounting for these recurrences, the authors considered the oncological results acceptable. In GRECCAR 2 the randomization and inclusion of clinical $\mathrm{N}+$ patients allowed the authors to detail the percentage of patients with positive lymph nodes in the TME specimens. The found no positive nodes in ypTO$1,8 \%$ in ypT2 and $40 \%$ in ypT3. Only one in 55 patients cNO had pathological positive lymph node. This led the authors to state that probably ypT2 cNO tumors might not benefit from completion TME because the probability of positive lymph nodes is very low. This leads us to consider that follow-up in ypT2 patients in oncologically sound.

By including ypT2 patients in the follow-up group the proportion of patients needing completion TME decreases drastically to between 9 and 19\%. In GRECCAR 2 this would mean that 3 in 4 patients that were supposed to have a completion TME would not have it. Only ypT3, R1 or N+ patients would need further surgery. By using completion TME in fewer patients the morbidity profile changes in favor of the local excision group. 


\section{Watch and Wait}

The percentage of patients complete pathologic response ranges from 8 to $24 \%$ (10). Local excision after neoadjuvant treatment studies showed a rate of complete pathologic response ranging from 27 to $49 \%$. These results suggest that the patients recruited for these studies were better responders than average patients. The selection of earlier rectal cancer contributes to this as the response rate seems to be related to the stage. The probability of complete pathologic response is $58 \%$ in $\mathrm{T} 1,28 \%$ in $\mathrm{T} 2,16 \%$ in T3 and $12 \%$ in T4 (10). In GRECCAR 2, $61 \%$ of the patients achieved a good response (ypT0-1), which was higher in CT2 than in CT3 patients with a good response ratio of 69 vs 51\% respectively. Small tumors like the ones recruited in these trials respond better to chemoradiotherapy and are the best candidates for an organ preservation strategy.

In these studies, complete clinical response was treated with local excision. These patients could have benefited from a watch and wait program. Local excision in this setting confirms absence of tumor in bowel wall but unfortunately cannot confirm nodal status to affirm complete pathologic response. Up to 4 $\%$ of patients have a local nodal recurrence after clinical complete response and watch and wait (11). Confirming ypTO disease might not be relevant, even more so considering that there is morbidity associated with local excision after neoadjuvant treatment. Local excision might prevent local regrowth, which occurs in $15,7 \%$ of watch and wait patients (12). Usually regrowth can be managed by salvage surgery $(12,13)$.

The strategy of local excision after neoadjuvant treatment seems to be best employed when dealing with a near complete response after an extended observation period. Some of these patients will have a complete pathologic response and others will fit the criteria established by these studies of disease downstaging with comparable oncologic long-term results with TME.

Two distinct groups of patients can achieve complete or near complete clinical response. The traditional patients that have neoadjuvant chemoradiation with locally advanced rectal cancer and this new group of patients with CT2NO which current guidelines only recommend radical surgical treatment. This second group responds better to the neoadjuvant treatments; however, non-responders will be over-treated. They will still receive radical surgery and receive chemoradiation with its consequences.

\section{Patient selection}

Considering the good results regarding morbidity and oncologic profile and the high rates of morbidity of completion TME, the best candidates for this approach are small tumors $(<4 \mathrm{~cm})$ in which local excision is feasible and are staged CT2-3 NO or N1. Small tumors respond better to neoadjuvant treatments. These patients should be restaged and if considered good clinical responders by the criteria of GRECCAR 2 study it is safe to proceed with local excision of the tumor. If the final pathology is ypT0-2, follow-up without completion TME should be offered to the patient as further surgery would increase greatly the risk important morbidity and the probability of residual disease in the mesorectum is very low.

\section{Functional outcomes}

Stijns (14) reported on the functional results of patients with successful organ preservation in the CARTS study. They found that $50 \%$ of the patients had major LARS. The combination of local excision and radiotherapy might explain these results as both can contribute do this syndrome.

Quality of life results were also published by Lezoche's group, they found that even though local excision patients reported better results in the early post-operative period at three years there was no difference in the scores evaluated between the local excision and TME groups (15).

\section{CONCLUSION}

Local excision after neoadjuvant chemoradiation achieves similar oncological results as radical surgery in highly selected patients with small rectal tumors. This strategy should probably be used in conjunction with watch and wait protocols. Patient selection is critical to decrease the proportion of patients in which completion TME is necessary because of it's increased morbidity.

\section{Conflicts of Interest and Source of Funding}

The authors declare there are no conflicts of interest. This article was not supported by funding.

\section{REFERENCES}

1. Glynne-Jones R, Wyrwicz L, Tiret E, Brown G, Rödel C, Cervantes A, et al. Rectal cancer: ESMO Clinical Practice Guidelines for diagnosis, 
treatment and follow-up. Ann Oncol Off $\mathrm{J}$ Eur Soc Med Oncol. 2017;28(suppl_4):iv22-40.

2. Habr-Gama A, Perez R0, Nadalin W, Sabbaga J, Ribeiro U, Silva e Sousa AH, et al. Operative versus nonoperative treatment for stage 0 distal rectal cancer following chemoradiation therapy: long-term results. Ann Surg. 2004;240(4):711-7; discussion 717-718.

3. Lezoche E, Baldarelli M, Lezoche G, Paganini AM, Gesuita R, Guerrieri M. Randomized clinical trial of endoluminal locoregional resection versus laparoscopic total mesorectal excision for T2 rectal cancer after neoadjuvant therapy. Br J Surg. 2012;99(9):1211-8.

4. Garcia-Aguilar J, Renfro LA, Chow OS, Shi Q, Carrero XW, Lynn PB, et al. Organ preservation for clinical T2NO distal rectal cancer using neoadjuvant chemoradiotherapy and local excision (ACOSOG Z6041): results of an open-label, single-arm, multi-institutional, phase 2 trial. Lancet Oncol. 2015;16(15):1537-46.

5. Verseveld M, de Graaf EJR, Verhoef C, van Meerten E, Punt CJA, de Hingh IHJT, et al. Chemoradiation therapy for rectal cancer in the distal rectum followed by organ-sparing transanal endoscopic microsurgery (CARTS study). Br J Surg. 2015;102(7):853-60.

6. Rullier E, Rouanet P, Tuech J-J, Valverde A, Lelong B, Rivoire M, et al. Organ preservation for rectal cancer (GRECCAR 2): a prospective, randomised, open-label, multicentre, phase 3 trial. Lancet Lond Engl. 2017;390(10093):469-79.

7. De Graaf EJR, Doornebosch PG, Tollenaar R a. EM, Meershoek-Klein Kranenbarg E, de Boer AC, Bekkering FC, et al. Transanal endoscopic microsurgery versus total mesorectal excision of $\mathrm{T} 1$ rectal adenocarcinomas with curative intention. Eur J Surg Oncol J Eur Soc Surg Oncol Br Assoc Surg Oncol. 2009;35(12):1280-5.

8. Perez RO, Habr-Gama A, Săo Juliăo GP, Proscurshim I, Scanavin Neto A, Gama-Rodrigues J. Transanal endoscopic microsurgery for residual rectal cancer after neoadjuvant chemoradiation therapy is associated with significant immediate pain and hospital readmission rates. Dis Colon Rectum. 2011;54(5):545-51.
9. Gérard J-P, Azria D, Gourgou-Bourgade S, Martel-Laffay I, Hennequin $C$, Etienne $P$-L, et al. Comparison of two neoadjuvant chemoradiotherapy regimens for locally advanced rectal cancer: results of the phase III trial ACCORD 12/0405-Prodige 2. J Clin Oncol Off J Am Soc Clin Oncol. 2010;28(10):1638-44.

10. Maas M, Nelemans PJ, Valentini V, Das P, Rödel C, Kuo L-J, et al. Long-term outcome in patients with a pathological complete response after chemoradiation for rectal cancer: a pooled analysis of individual patient data. Lancet Oncol. 2010;11(9):835-44.

11. van der Valk MJM, Hilling DE, Bastiaannet E, Meershoek-Klein Kranenbarg E, Beets GL, Figueiredo NL, et al. Long-term outcomes of clinical complete responders after neoadjuvant treatment for rectal cancer in the International Watch \& Wait Database (IWWD): an international multicentre registry study. Lancet Lond Engl. 2018; 391(10139):2537-45.

12. Dossa F, Chesney TR, Acuna SA, Baxter NN. A watch-and-wait approach for locally advanced rectal cancer after a clinical complete response following neoadjuvant chemoradiation: a systematic review and meta-analysis. Lancet Gastroenterol Hepatol. 2017;2(7): $501-13$.

13. Kong JC, Guerra GR, Warrier SK, Ramsay RG, Heriot AG. Outcome and Salvage Surgery Following 'Watch and Wait' for Rectal Cancer after Neoadjuvant Therapy: A Systematic Review. Dis Colon Rectum. 2017;60(3):335-45.

14. Stijns RCH, de Graaf EJR, Punt CJA, Nagtegaal ID, Nuyttens JJME, van Meerten E, et al. Long-term Oncological and Functional Outcomes of Chemoradiotherapy Followed by Organ-Sparing Transanal Endoscopic Microsurgery for Distal Rectal Cancer: The CARTS Study. JAMA Surg. 2019;154(1):47-54.

15. D'Ambrosio G, Picchetto A, Campo S, Palma R, Panetta C, De Laurentis $F$, et al. Quality of life in patients with loco-regional rectal cancer after ELRR by TEM versus VLS TME after nChRT: long-term results. Surg Endosc. 2019;33(3):941-8. 